\title{
An analysis of screen reader use in India
}

\author{
Ted McCarthy ${ }^{1}$, Joyojeet Pal ${ }^{1}$, Edward Cutrell ${ }^{2}$, Tanvi Marballi ${ }^{3}$ \\ ${ }^{1}$ University of Michigan \\ School of Information \\ Ann Arbor, MI 48104 \\ \{epmccart, joyojeet\}@umich.edu \\ ${ }^{2}$ Microsoft Research India \\ Technology for Emerging Markets \\ Bangalore, India 560025 \\ cutrell@microsoft.com \\ ${ }^{3}$ New York University \\ Polytechnic Institute \\ Brooklyn, NY 11201 \\ tanvimarballi@gmail.com
}

\begin{abstract}
We present the results of two surveys and a qualitative interviewbased study with users of screen readers in India. Our early interviews moved us in the direction of examining patterns that differentiate users of two particular software applications - the dominant market standard JAWS and the free, open source challenger NVDA. A comparison between the two is timely and particularly relevant to issues elsewhere in the developing world. In the short term, the question of choosing one application over another could be based on price and support for custom-made applications, but in the long term, issues of language support are likely to be of concern as well. We explore software adoption behavior and present results that show the relationship between the quality of audio and peoples' willingness to use one software over another. We also compare the switch from JAWS to NVDA to other kinds of switches from dominant software to open source options. In conclusion, we discuss the business aspects of screen readers and examine why the comparison between these two applications is particularly important in the discussion on accessible personal computing for people with vision impairments in the developing world.
\end{abstract}

\section{Categories and Subject Descriptors}

K.4.2 [Computers and Society]: Social Issues - Handicapped persons/special needs.

\section{General Terms}

Design, Economics

\section{Keywords}

Disability, ICTD, Assistive Technology, Screen Readers, Open Source Software, JAWS, NVDA

Permission to make digital or hard copies of part or all of this work for personal or classroom use is granted without fee provided that copies are not made or distributed for profit or commercial advantage and that copies bear this notice and the full citation on the first page. Copyrights for components of this work owned by others than ACM must be honored. Abstracting with credit is permitted. To copy otherwise, to republish, to post on servers or to redistribute to lists, requires prior specific permission and/or a fee.

ICTD '12, March 12 - 15 2012, Atlanta, GA, USA

Copyright 2012 ACM 978-1-4503-1045-1/12/03 ...\$10.00.

\section{INTRODUCTION}

Approximately $90 \%$ of the world's visually impaired live in the developing world ${ }^{1}$, and an estimated 15 million Indians are visually impaired. ${ }^{2}$ For people with vision impairments, access to Assistive Technology (AT) can be decisive for participation in a modern labor force where technology is increasingly ubiquitous. Since most AT for people with vision impairments tends to be produced in the industrialized world, primarily for users from those countries, there are problems of price point, support infrastructure, and language regionalization that can be hurdles for AT adoption in the developing world. As a result, screen readers that are free or low-cost and easily extensible to locally relevant software needs are of importance to the needs of the developing world.

Although some work has looked at the importance of low-cost AT for the needs of the developing world [1], there has been little systematic investigation of the actual mechanics of low-cost options to dominant (and high-cost) software. Furthermore, there are few empirical studies that present data on the state of AT use for people with vision impairments in any part of the developing world. Despite the large community of persons with vision impairments, their relatively small size as a 'market' for AT products has limited the amount of existing research on the technology use of this community.

In this paper, we explore the use of screen reader software by people with vision impairments in India, and specifically examine their behavior related to low-cost options on screen reading technology. Screen reading software refers to programs that enable blind or visually impaired users to better operate a computer; they essentially replaces much of the graphical user interface, allowing users to interact fully with a computer using the mouse and receiving audio feedback (or tactile, if the computer if equipped with a Braille output device - however, none of our participants reported using a tactile output). The screen reader employs a text-to-speech engine (TTS) that "reads" out text from the screen, notifies users of the applications they are using, their location within a spreadsheet or web page, etc. The quality of these TTS "voices" varies significantly, from very human-like voices to voices that sound quite synthesized.

\section{APPROACH}

We use a mixed-methods approach for the empirical data presented here with input from a total of 200 respondents. In

\footnotetext{
${ }^{1}$ http://www.who.int/mediacentre/factsheets/fs282/en/

${ }^{2}$ http://www.disabilityindia.org/djfactsoct07D.cfm
} 
addition to an extensive review of the existing literature on screen reading technology, we studied forums of users and developers for screen readers, with specific attention to two particular software programs - JAWS (Job Access with Speech) and NVDA (Non Visual Desktop Access) - which, between the two, have the highest installation rates among people with vision impairments ${ }^{3}$.

Following our study of secondary data, we conducted in-depth qualitative interviews of 20 users of screen readers, and those interviews were used to create two surveys; one specifically surveying screen reader use, and another on open source software use.

The first survey examined a number of issues around screen reading technology use such as individual preference for one screen reader over another, online activity, use of TTS, discussions of experience and typical problems with various screen readers, and ability to complete specific tasks using screen reading technology. The survey was conducted in-person in two cities of India - Mumbai and Bangalore, where members of the team were located - or online. Online respondents were reached through web-based forums on Inclusive Planet and Access India, the first a social networking site and the second a mailing list, both for persons with vision impairments in India. The survey was fully accessible and could be completed using an online or desktop-based screen reading application - roughly a third of respondents completed the survey online, and the remainder were surveyed in person. All surveys were conducted in English.

The second survey was very specifically aimed at sampling behavior related to the switch from a dominant proprietary software product to an open-source product. The decision to conduct this survey came from early interviews in which we found a sizable sampled population discussing an interest in moving from JAWS, the dominant screen reading software, to NVDA, an open source program and relative newcomer with a comparatively smaller but rapidly growing market share. In this survey, our goal was to understand economic versus ideological motivations in switching to open source software products generally to see what comparisons could be drawn to screen reading software in particular. This survey was conducted via mailing lists of open source software user mailing lists.

Ideally, we would have compared the results of the screen reading survey with a pre-existing survey of open source product use, but none such to our knowledge exists in India. Thus, the second survey was performed with the purpose of fulfilling an important gap in the literature.

There were 101 users sampled for the screen reading software survey. The median age for respondents of this survey was 26 , and the average number of years of screen reader use was $5.4 .80 \%$ of respondents own a screen reader for their computer, and $75 \%$ of those surveyed use a screen reader on their mobile phones. About half $-48 \%$ - of users who own screen readers reported that they had obtained a pirated version, but it seems likely that this may be a conservative estimate, though an attempt was made to ensure the confidentiality of respondents' answers. There were 99 users sampled for the open source software, $40 \%$ of whom were Indian and $60 \%$ of whom lived elsewhere. Our sample was heavily male, with that gender comprising $79 \%$ of the respondents.

\footnotetext{
${ }^{3}$ http://webaim.org/projects/screenreadersurvey3/
}

Our efforts were primarily invested in sampling screen reader users for two reasons - first, we see the open source survey as mainly playing a supporting role in explaining one of the various aspects relating to screen reader software preference in India, and secondly, the population of screen reader users in India is fairly scattered (even in these two cities) and to sample a significant enough population of assistive technology users was what we saw as the critical task ahead of us. There are a few biases in the populations sampled - for instance, sampling the open source software-using community through mailing lists dedicated to that community likely biased us toward users who have a greater ideological motivation in their choice of technology. However, because the survey compares application use across various kinds of open source software (operating systems, graphics applications, word processing applications etc.), we can control for this within the responses.

One important aspect of the survey was the distinction between the locations of the interviewees based on the kind of survey being conducted. In the screen reading survey, all the respondents were based in India, although some of the experts recruited for the in-depth open-ended interviews were located outside of the country. For the open source software survey, we wanted to get a sampling of non-Indian users as well as Indian users. The reason for this distinction, besides our need to focus on Indian users, was that the process of procurement of and training in screen reading software differs in various parts of the world - so access to expensive screen reading software like JAWS (at about $\$ 1000$ per license) is mandated by various governments for both home and workplace use due to local disability-related laws. In contrast, use of most other software typically requires an investment by the user (irrespective of piracy), and so the same problem was not applicable to the open source survey.

\subsection{Sample Description}

Both the open source software and the screen reading survey population were not random, therefore it is not clear to what extent our sample reflects the rest of the population in that category. For the screen reader survey, $34 \%$ of respondents were female and $66 \%$ were male, while for the open source survey, $29 \%$ of respondents were female and $71 \%$ were male.

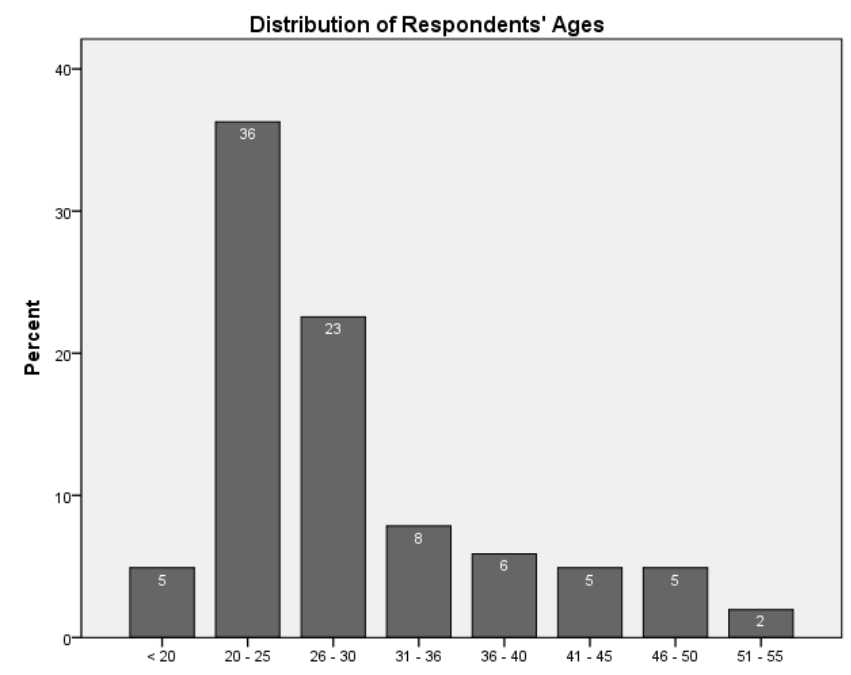

Figure 1: Age distribution of Screen Reader survey respondents $(\mathbf{n}=101)$ 


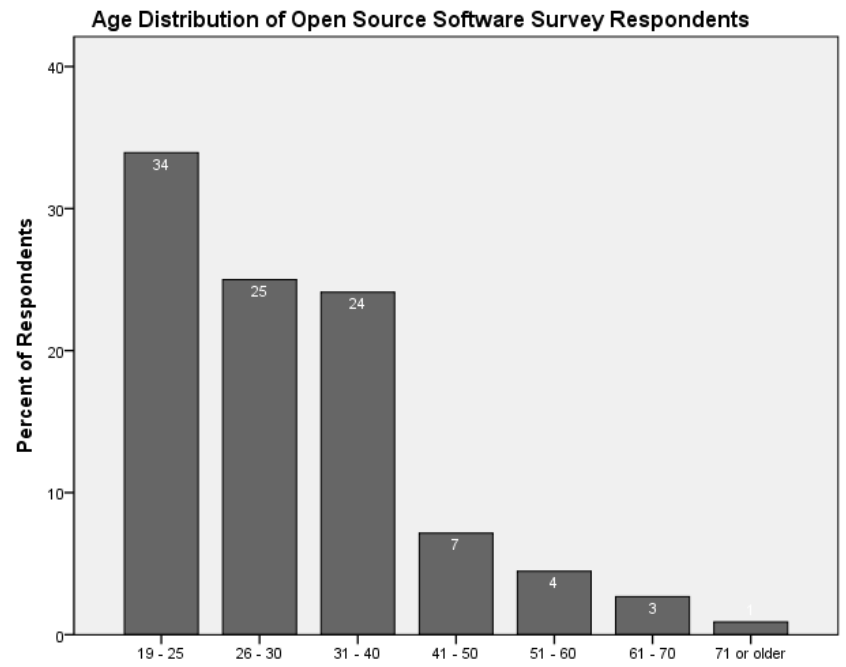

Figure 2: Age distribution of open Source survey respondents $(\mathbf{n}=99)$

The age distribution of the respondents in both surveys skews relatively young, as can be seen in figures 1 and 2 . One important difference between the screen reader survey and the open source survey is that the open source survey has a much higher proportion of younger, probably college-going or late school respondents, whereas the screen reader-using population tended to be a few years past college, either late in their computer training or early in their careers.

If we look at the education levels of the respondents, we find again that both populations are very highly educated, with a majority possessing at least a bachelor's degree.

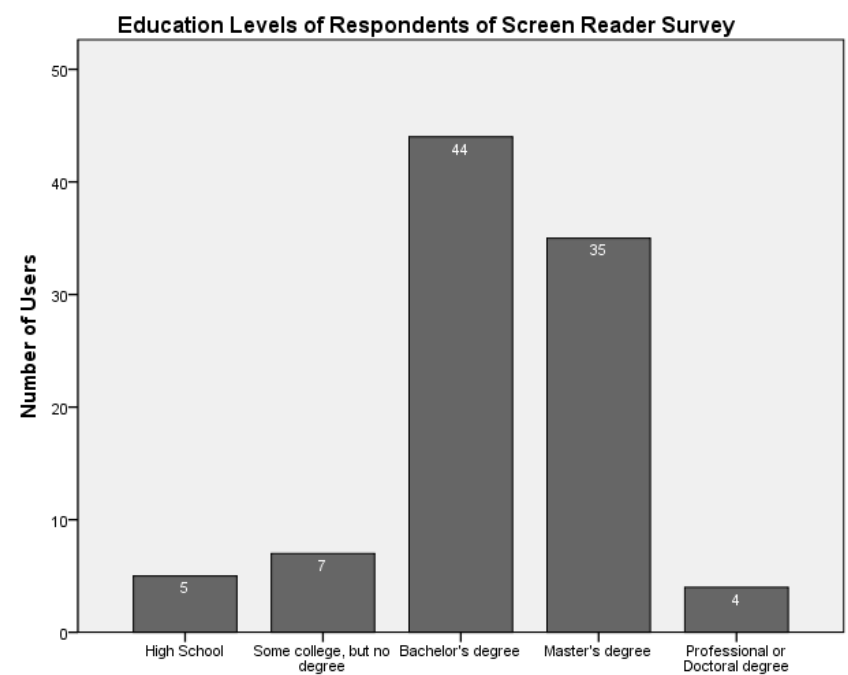

Figure 3: Education level of screen reader survey respondents

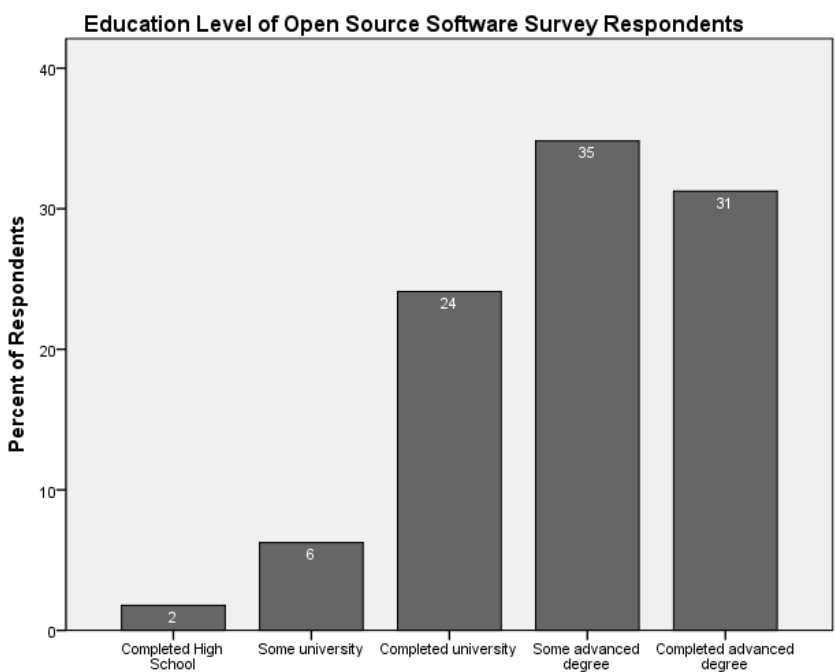

Figure 4: Education level of open source survey respondents

In the case of open source survey respondents, this distribution does not matter significantly, though in the case of screen reader users, the skew is indicative of the fact that the population is not a good representation of vision-impaired persons in India more generally.

In the results presented, we do not name any of the interviewees who offered quotes, except those who are public figures and/or disability rights activists, and who agreed to allow their names to be published.

\section{ANALYSIS OF RESULTS}

The results from the surveys and interviews show that although screen readers still form a fairly small market in India, there is a fairly active community of users which largely show use patterns comparable to some of the major global trends. We discuss here some of the key findings from the surveys, and interpret them in the context of qualitative research with assistive technology users in Mumbai and Bangalore.

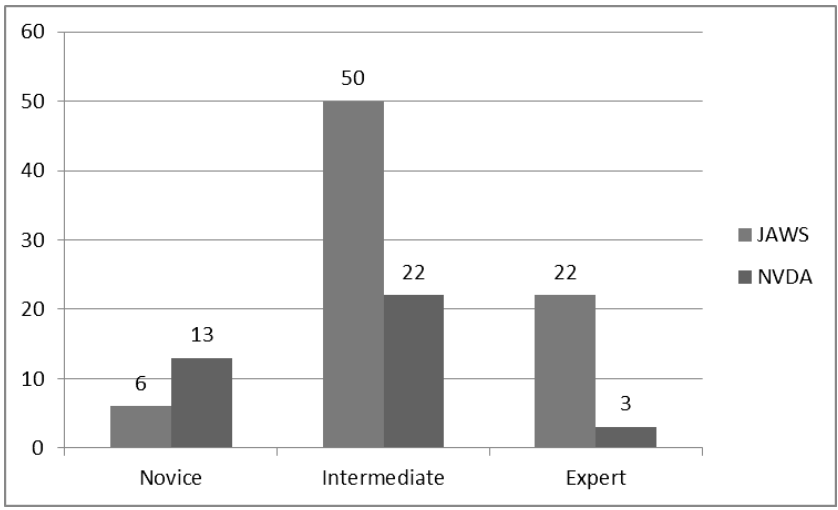

Figure 5: Level of expertise on screen reading software

We found that JAWS and NVDA were by far the most commonly used applications -85 of the total 101 respondents used JAWS regularly, while 40 used NVDA regularly. WindowEyes was the closest third at 6 users, making the battle for the Indian market pretty much a two-way competition between these two 
applications. However, we see that a proportionally higher number of users were intermediate or expert at JAWS than they were at NVDA, indicating that many NVDA users tended to be casual or experimental users.

\subsection{Predominance of pirated JAWS}

Despite its high cost, JAWS is practically ubiquitous among computer-using populations with vision impairments in India. In the past it was common to come across people who used trial versions of JAWS because of the unaffordable licensed version, but due to the relatively high degree of software piracy in the country, it is fairly trivial to obtain a copy of JAWS at an extremely low cost or completely free.

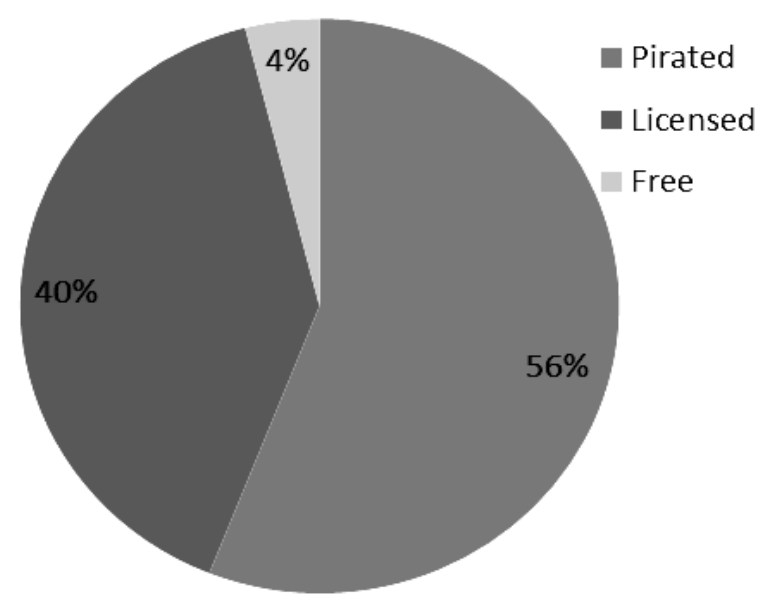

Figure 6: Installed software (\%) at place of primary use

As we see in figure 6 above, over half the population stated that their primary screen reader was pirated. This of course does not imply that the remaining $40 \%$ actually purchase their software, it simply means it is a licensed copy - which could be a copy at a place of work, a donated copy, or for that matter a licensed copy of an open source software application (if they chose not to classify that as free). When we asked users how many of them had made a paid purchase of their screen reader, it was just $11 \%$ of the sample, implying that the culture of acquiring paid versions of screen reading software is not very prevalent.

"Pirated JAWS is available so freely out here [in India]. Very easily. So, it's nothing to be proud of, but still, it's a fact of life that it's very freely available. Hence people will not go into NVDA."

-HM, Screen reader user, discussing piracy Mumbai

Indeed, at roughly $\$ 1000$ a license, just slightly below the 2010 estimate of the average Indian's per capita annual income, the odds of a large number of people willing to pay for JAWS is on the unlikely side. Of at least equal importance is the lack of a significant social deterrent to pirating screen reading software. This is evident in the approach of one important social movement of people with vision impairments in 2010, the 'Right to Read' campaign in India. It took the normative position that since Indians had the right to read, but the government nonetheless did nothing to provide print-impaired people with this right, the country's intellectual property laws were at odds with the disabled population's 'Right to Read.'

\begin{abstract}
"We have the right to read, but less than 5\% of the materials are available in accessible formats. What is the right then? We will pirate the material; let us see who wants to sue this group."
\end{abstract}

-Right to Read activist

Software piracy was at roughly $64 \%$ in India in $2010^{4}$, and Osorio has found that social beliefs around piracy can increase its prevalence in a country [2]. The same research also finds that illegal copying increases with high software price and lower GDP, alongside which other research shows that software companies operating in markets with a high degree of piracy have limited incentive to build tools for those markets [3]. In short, there exists a situation wherein JAWS is easily pirated, and the developer of the software has no real incentive to try to reach the Indian markets in the short term. The high prices of the software are in part due to the structure of the industry, with few makers building highly sophisticated software for a relatively small audience of users, but more importantly, the software is frequently sold to institutional buyers or governments in countries that have disability laws. Thus the dependence on the end consumer's ability to pay is limited.

JAWS has a history of dominance within the screen reading market for more than the past decade, and the same applies within India. The establishment of early adopters of screen readers in India quickly moved to JAWS, leading to most computer training classes for screen reading to likewise teach JAWS. In the current scenario, the switch away from JAWS has often been driven both by individuals motivated by an ideological preference for open source screen readers and by companies unwilling to use pirated software for their vision impaired employees, which are then interested in finding workable alternatives.

\subsection{Significant effects of 'surface' factors}

One of the most significant outcomes of the survey was the relationship of TTS "voice" with the preference for a particular software. We were surprised in initial interviews to find how many people dropped NVDA very soon after their first attempt at using it because of the audio "voice" quality of the TTS engine.

"The Eloquence [TTS] that comes default with [JAWS]
is very simple to hear, people get used to it very fast."

-Dr. Homiyar Mobedji, Physiotherapist and screen reader user, Mumbai

"If you're going to be listening to [a mechanical voice] ten hours a day, it's tough."

Nirmita Narasimhan Lawyer and screen reader user, Bangalore

\footnotetext{
${ }^{4}$ http://portal.bsa.org/globalpiracy2010/downloads/press/pr_india. pdf
} 
Figure 8: User preferences for screen reader features

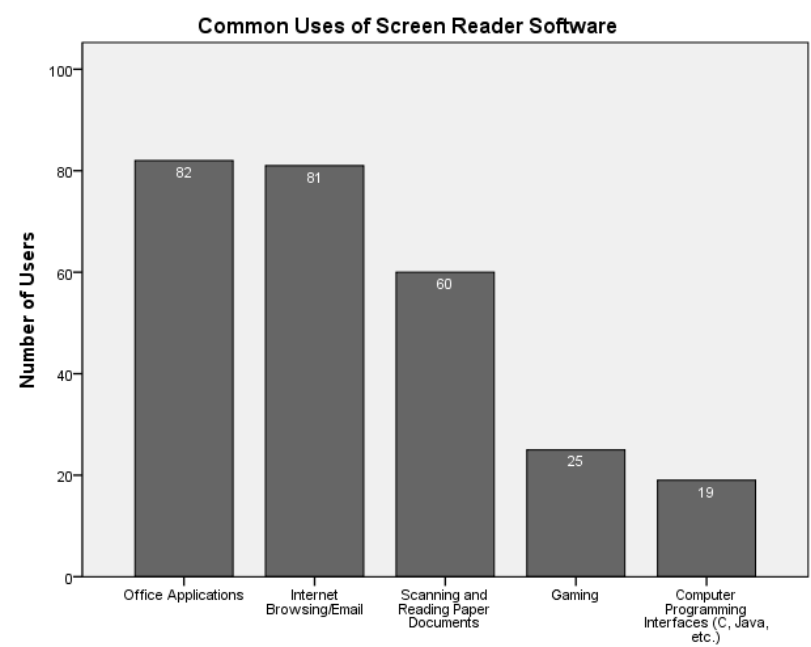

Figure 7: Common uses of screen reader software

And while respondents clearly marked their preference for screen readers with a natural voice (such as JAWS), the data about their preferences after becoming more experienced, and discussions with advanced screen reader users, suggests something rather different. The survey data shows that as users get more experienced with screen reader use, they speed up the audio output to the point where it sounds very much like a mechanical voice. In fact, some advanced users specifically stated preferring a mechanical voice for the standardized intonation.

As a user becomes an advanced screen reader user, the output voice seems to matter less than the number of applications supported. The results of the survey show strongly $(p=.010)$ that advanced users find application support to be the most important quality in a screen reader, while novice users placed the most importance on the voice quality of the text-to-speech engine used by the software.

Shown visually, the survey of screen reading suggests an inverse relationship between level of expertise and valuation of features, as seen in figure 8 .

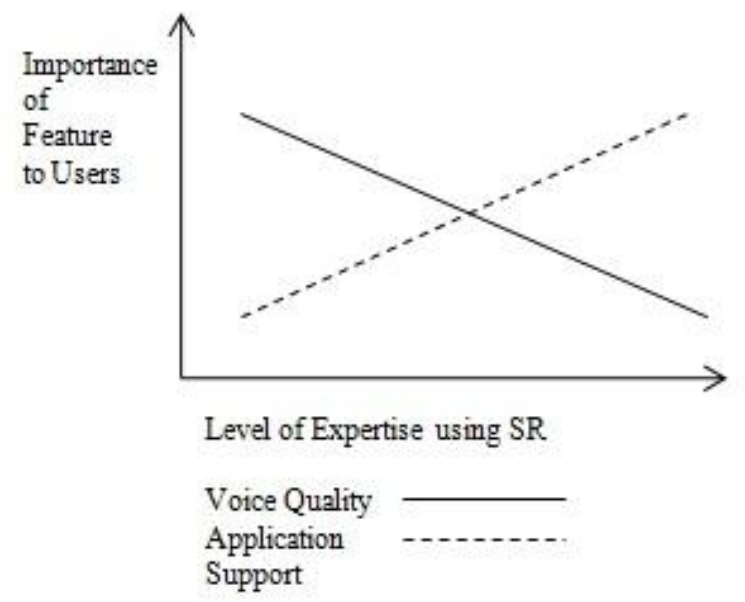

We find strong statistical significance when looking at the figures for preference for application support over voice quality as screen reader users move from novice to advanced.

Much as we may find that the more one uses a screen reader, the comparatively less one cares for the quality of voice, the initial adoption factor makes an important impact. The importance of voice quality for early stage users cannot be overstated. One important factor reinforcing the preference for JAWS is cellular phone users' familiarity with the output.

Talks uses Eloquence. And most people in India are very familiar with Eloquence, hence Talks is very readily accepted compared with MobileSpeak... if you are given a choice of pirated softwares of Mobile Speak and Talks, people would prefer Talks, in India."

-HM, Screen reader user Mumbai

Thus the fact that that Talks is the dominant cellular phone-based screen reading application in India (used by $98 \%$ of our respondents) further strengthens the preference for Eloquence (the natural-voice speech synthesizer used by JAWS), and as users were unwilling to try out the cellular alternative MobileSpeak, they similarly dismissed NVDA because of the voice.

Up until recently, people have been trained using only JAWS or at the very least 'primarily' JAWS at training centers, and as $98 \%$ of users surveyed cited JAWS as the one screen readers they first used (one learned using NVDA and one using NVDA and JAWS), they will likely continue to use this software well into more advanced stages. Thus, even after one has ceased to value the quality of voice, the loss in efficiency from switching to a new piece of software and re-learning a new interface often prevents this from occurring.

\begin{abstract}
"Since it's existed for quite some time, people are actually addicted with the Eloquence synthesizer, because the quality of the speech is pretty good... So, now if you ask them to transition from that speech quality to a little bad speech quality [as in the case of NVDA], it's actually the mindset issue, they cannot really change it. It takes time for them to change. That's the problem. They simply say that NVDA is bad just because of the speech quality."
\end{abstract}

Srinivasu Chakravarthula, Yahoo! accessibility consultant, Bangalore

This can be seen as comparable to the behavior of people switching from perhaps Windows to Linux or PC to Mac, wherein there is a learning curve with which some users are willing to experiment, but with the knowledge that there may be some efficiency loss at least initially. More importantly, the lesson here is that institutional factors for software adoption are probably critical - so while a user may not ordinarily be willing to try out an alternative to a certain piece of software, when thrown into an institution which primarily supports such an alternative, the user must adapt. Our survey shows that NVDA clearly follows JAWS 
as the second-choice software and the software users are most likely to rate as being able to switch to if needed.

\subsection{Cost and software switching}

The range of factors influencing behavior relating to switching from the dominant 'industry standard' software of JAWS to lower cost alternatives bear an interesting comparison to other comparable switches. The results of the open source software use survey give us interesting insights into some of the influencing factors.

Table 1: Emphasis on cost as a factor for switching to an Open Source technology

\begin{tabular}{|l|l|l|}
\hline Application type & $\begin{array}{l}\text { Mean } \\
\text { Emphasis on } \\
\text { Cost }\end{array}$ & $\begin{array}{l}\text { Difference in } \\
\text { cost emphasis } \\
\text { from that } \\
\text { placed on OS }\end{array}$ \\
\hline Operating System & 3.96 & N/A \\
\hline All non-OS & 3.39 & $\mathrm{p}=.001^{* * *}$ \\
\hline Office Suite & 3.14 & $\mathrm{p}<.001^{* * *}$ \\
\hline Photo Editor & 3.25 & $\mathrm{p}<.01^{* *}$ \\
\hline PDF Editor & 3.23 & $\mathrm{p}<.01^{* *}$ \\
\hline Audio Editor & 3.54 & $\mathrm{p}=.10$ \\
\hline Statistical Software & 3.75 & $\mathrm{p}=.47$ \\
\hline Video Editor & 4.03 & $\mathrm{p}=.80$ \\
\hline
\end{tabular}

$* * *=$ significant at $\mathrm{p}=.001 ; * *=$ significant at $\mathrm{p}=.01$

Table 1 shows the results of participant rating of cost as a factor in choosing open source rather than commercial software, rating emphasis on a scale from 1 to 5 . A score of 1 indicated that cost was a highly significant factor in choosing the open source product, and a 5 indicated that cost was of no matter in choosing the open source software over a commercial approximate alternative. The results show that there is much greater price sensitivity for office applications, document management systems, and graphical development applications compared with operating systems in the decision to 'switch.' Thus, the high cost of dominant software options in those categories - namely Photoshop, Acrobat, or MS Office - are more likely to push a user towards a switch to a free version when compared with the switch between a commercial and open source operating system, especially when that version offers the most commonly used functionalities. In contrast, we found that proprietary specialized software, such as SPSS statistical software, is less driven by price.

We explain this as a factor of user expertise - thus document management or office application software can be qualified as generic, therefore used by a fairly wide number of users. In comparison, video editing software or statistical packages are not likely to be evaluated easily in a framework such as ours, because we find far fewer 'expert' users in a random sampling. That is to say, those users whose professions depend on SPSS or video editing software, and only those users, are likely to have much lower price sensitivity or impetus to switch to open source options, especially if their professions depend on it.

What is perhaps most interesting is that we found the Windows to Linux switch to be significantly less driven by cost than by other factors. There are those who are willing to 'experiment' with Linux as they have more than one computer, and these users tend to be more casual users, and another group that dedicatedly uses Linux either due to preference for the greater customizability, or even on ideological grounds. Our results show that users are significantly more likely to choose an open source application due to reasons related to cost if that application is not an operating system, especially if it is an office suite, photo editor, or PDF editor - that is, those applications which seemingly offer comparable services when found in open source when compared with their commercial equivalents.

The existence of a network of users is also fairly critical to the adoption of any new software. In the case of screen reader use, we found that the typical user frequently resorts to queries either from within one's immediate circle or through online forums. A lack of a sufficient network of users has been discussed elsewhere as a common barrier for software switching behavior [4], including specifically in the case of open source options to existing dominant software [5-7].

These same network effects can be seen in our sample of mobile phone screen reader users as well. In general, people were happy with Talks, and chose it either because it was the first mobile TTS they had heard of, or through networks of friends and colleagues. For most people the choice of Talks, especially of that over Mobile Speak, was often due to what was considered the market standard because of referrals by friends. Several users explicitly mentioned being comfortable with the voice quality of Talks, and said they were uninterested in switching.

For people with vision impairments, a screen reader functioning at a sub-optimal level, even briefly, can have an extremely adverse impact on productivity. In India, given a relatively unfriendly employment environment for people with disabilities, this is an even greater concern, because employees cannot take the risk of being unproductive, albeit for a short period of time. The 'cost' thus of the switch can be fairly significant.

"I tried [NVDA] once very briefly, but the problem for me is if I have to switch to another screen reader, effectively I need at least one week or ten days to familiarize myself with it and... for that period of time I'm not able to work productively because I'm still discovering these things"

NM, JAWS user, Bangalore

Several of our interviewees explicitly mentioned the importance of the large network of extant JAWS users, the extensive software documentation available for the product, and the general entrenchment of the software within the visually impaired community, all of which exist to a much lesser degree in the case of NVDA. The switch to NVDA (there were very few instances of any other open source software discussed by the users, so we restrict our analysis to this comparison) came with the fear of not finding the right support environment, especially if the work was mission-critical. 


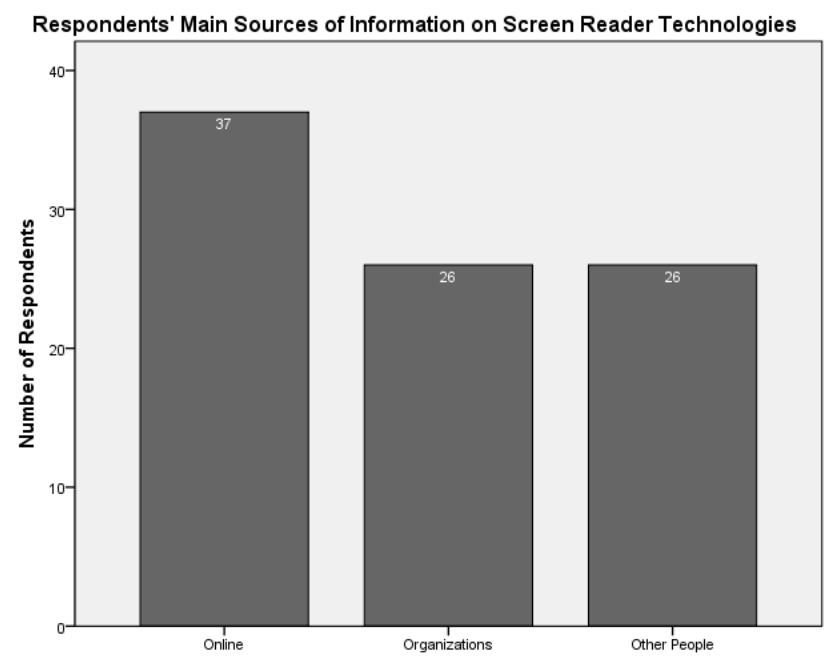

Figure 9: Top ranked sources of information on screen readers

A factor not immediately obvious on switching behavior is that social networks play an incredibly important role in vision impaired computer users' technology choices. This emerged somewhat in our interviews with people, but as we see with the top sources of information on technology in figure 9 above, online sources (typically social networking forums), organizations (typically NGOs) and others (typically friends) are top sources on issues relating to screen readers and AT. In other words, new technologies spread very quickly through word of mouth sources and social networks.

\subsection{The discourse of complexity}

One of the motivations behind exploring the parallels between JAWS/NVDA and perhaps the most easily comparable condition of Windows/Linux was the perception of complexity. As opposed to the Windows OS packaged with most off-the-shelf computers, there is typically an additional effort involved in switching to Linux. The perception that the average Linux user needs to be more technical is fairly pervasive, as the Linux OS is generally perceived as having a steep learning curve, associated with an early group of command line hackers [8]. This made it such that when user-friendly releases of Linux (e.g. Ubuntu) arose, there was a need to market these as usable by non-geeks [9].

This association with the hacker ethic has also contributed to the idea of open source software use as itself being tied to the intent of, or active participation in, the further development of applications [10] or at the very least in user groups [11]. Arguably, this 'geekification' plays a role in the idea that any open source software requires a greater ability to deal with complexity and the minutiae of software functionality.

To some extent, this was reflected in computer courses for visually impaired populations focusing only on JAWS, much in the way that introductory computer courses do not stray beyond Windows. The few computer courses that do offer training in NVDA (Enable India in Bangalore, for instance) offer it as secondary to JAWS, usually spending very little time on it.
"Perhaps the largest barrier to NVDA use is that eSpeak [the default TTS for NVDA] is not being introduced at the training center level"

Dipendra Manocha, Screen reader user and disability rights activist, Mumbai

In our own sample as well, JAWS was clearly the dominant preference ( $100 \%$ of the screen reading sample, in fact). While the NVDA users were far outnumbered by the JAWS users, the NVDA users were also slightly more technical. More than a third of the high-frequency NVDA users (5 out of 14) wrote scripts for their screen readers, whereas just about a fifth (6 out of 29) of the high frequency JAWS users from our sample were writing scripts. The results are not statistically significant, but nonetheless underline the notion that feeds the idea that one needs to be more technical to be an NVDA user.

\subsection{Screen reading in the work environment}

\begin{abstract}
"Middle-level and startup companies... cannot afford to spend about 1000 dollars [per license of JAWS] ... If people start using open source screen readers like NVDA, it's easy to convince an employer to give an employment opportunity to these candidates, and it's easy for them to get in onto the payrolls... I recommend our trainees to learn using NVDA because it's easy for us to generate employment for them."
\end{abstract}

Srinivasu Chakravarthula, Yahoo! accessibility consultant, Bangalore

By 2010, Indian software companies had started investing in developing NVDA for compatibility with their internal systems. This is an important direction for the future of screen reading as it indicates a recognition among companies that licenses for expensive screen reading software may not be viable in the longrun. With the implementation of the United Nations Convention on the Rights of Persons with Disabilities and India's own disability-related legislation, it is clear that larger corporations are likely to see an increase in employees with vision impairments. Alongside this, the increasing implementation of intellectual property laws in the organized sector has meant that 'Pirated JAWS' is not a serious option for the workplace, even if employers choose to turn a blind eye to whatever is installed on their employees' personal machines. Thus stable versions of NVDA that can handle custom-made internal applications are likely to grow in prominence among major employers.

"People aren't seeing the large picture - eventually they're going to have to pay for [pirated versions of JAWS] - if they get a job, or... whether out of their own pocket, or through a company, or something else."

Dipendra Manocha, Screen reader user and disability rights activist, Mumbai

\subsection{Regional Language and Accent Support}

Though there are many millions of speakers of the 22 official languages of India, this distribution of speakers is not proportionately represented in the digital realm [12]. Though this 
may be due in part to the fact that wealthier Indians tend to have a higher command of English, further research is necessary to definitively conclude to what extent this is the case. Nevertheless, the development of TTS engines in lesser-spoken languages has piqued the interest of the research community [13] [14] as well as a great number of organizations, activists and individuals in the vision impairment community in India.

'I'd be quite happy to switch to another screen reader with an Indian language TTS once that gets up and working"

$\mathrm{NM}$, screen reader user, Bangalore

Within India itself, a great number of NGOs and government organizations are working toward the development of TTS for smaller languages. In the past, the DAISY Forum India has partnered with local NGOs to develop local-language support, and an agreement was recently made to do the same for the development of several more languages, including Gujarati and Marathi. Additionally, the TTS Consortium, a research group consisting of several IITs (Indian Institute of Technology) from different cities as well as C-DAC (Centre for Development and Advanced Computing), a government-funded research group, is currently involved in a project to develop a number of regionallanguage TTS. ${ }^{5}$

While text-to-speech engines exist for a number of Indian languages, these are often of poor quality or too expensive for most users. Preference for Indian English accent TTS among Indians (and particularly that matching the subjects' local accent) is higher when compared with a US English accent TTS, the accent most readily available, and therefore most commonly used, in screen readers in India. Additionally, findings show a decrease in intelligibility for those users with worse English when using the US English accent TTS [14]. This points to an especial need for high-quality local language or Indian English-accent TTS for those with poorer English, which likely includes many members of the visually impaired community in India, given the correspondence between that population and higher rates of poverty in some parts of the developing world.

The results of our own survey also showed a desire for regional language TTS development in India - 52\% of respondents indicated that there is some language or accent in which they would rather use their screen readers, and these answers were spread among 8 different Indian languages, as well as the Indian English accent. With the realization that $98 \%$ of respondents indicated that they primarily use a screen reader with either American or British English, and only one user regularly utilizes a screen reader with an Indian English accent, it seems that there is indeed a desire within the visually impaired community in India for high-quality TTS in local languages.

"So, in the first act, people are forced to use screen reader English, for English screen reader. And when it comes to a specific reading purpose, there is where they

\footnotetext{
${ }^{5}$ http://www.cdacmumbai.in/index.php/research_and_publications /projects/text_to_speech_synthesis_systems_for_indian_langua ges tts il
}

want it in Indian language... there's a demand. Growing demand, really really growing demand for this [Indian language screen readers]."

$\mathrm{SN}$, screen reader trainer, Bangalore

Indeed, "high-quality" TTS for local languages brings up an important distinction: it is not enough to merely build a TTS that works for smaller languages, but this TTS must be usable, or else people will fall back on using one of the more established TTS, even if it is not developed in their preferred language. Tucker and Shalonova show that, while the implementation of a functional local-language TTS can be easily undertaken, particularly with open source TTS such as eSpeak and Festival, it takes significant time and expertise to produce a local-language TTS of high enough quality such that it is likely to be successfully used [13].

Findings from our survey support this: in fact, all 9 of the languages or accents participants said they would prefer to use over their current TTS already exist. Of the 22 respondents who had used a TTS in their preferred language, but did not regularly do so, $14(64 \%)$ responded that they did not use this TTS because of its insufficient quality, whether due to poor "voice" quality or because of insufficient synthesizer speed or performance. Additionally, of those 13 who provided a reason as to why they had not tried a TTS with the language of their preference, 11 respondents stated that they had been unable to gain access to this TTS, often stating (incorrectly) that TTS for this language does not exist, that it was because they did not know where to find the software, or even because they were unable to figure out how to install the TTS or make the proper settings change to enable their screen readers to utilize the TTS. While this is a very small sample $(\mathrm{N}=13)$, the fact that 11 of the 13 users who stated that they had not used a TTS language of their preference listed a reason other than not wanting to use it (even when most of those respondents knew that the TTS existed) shows that there is much to be done to increase access to regional language TTS besides simple development. Users must be made aware of the existence of these TTS, and must be able to access and easily use them as well.

Taking all these findings into consideration, it appears that the mere development of a "functional" TTS for small languages is not enough; these TTS must be of high quality, well-marketed, and easily accessible, both from the standpoint of obtaining the software and from that of actually using it.

\section{"There are no standards for Indian languages... there is no standard keyboard layout."}

$\mathrm{SM}$, former SAFA developer and screen reader consultant, Bangalore

While there is evident desire among the visually impaired community in India for local language TTS and screen reading software, there are a number of obstacles presented by this challenge that are likely not immediately apparent, even once the difficulties presented by the development of high-quality TTS are addressed. For instance, the lack of standardization in Indian computer hardware came up as a problem in several interviews with developers who had been involved in the SAFA (Screen Access For All) project to develop an Indian-language screen reader. Among other complaints, one that was mentioned 
numerous times was the number of computer keyboard layouts used for Hindi alone: developers interviewed described the number of Hindi keyboard layouts as lying somewhere between 15 and 21 different configurations, all of which would require individual attention to be fully supported by the screen reader software. (The SAFA project settled on developing support for three of these.)

Additionally, the concern over the general lack of Indian language screen reader-friendly fonts (namely Unicode) was brought up often. Though most screen readers are written to support Unicode, many websites, including popular Tamil and Hindi news sites such as Eenadu and Jagran, use ASCII fonts, and therefore remain inaccessible, even to most screen readers that support these languages [15].

\section{"Most TTS' support Unicode based fonts. However, fonts in Indian languages are older than Unicode, and many of the popular publishing industry fonts are still not following Unicode coding system."}

$\mathrm{DM}$, former SAFA developer, Bangalore

Though a report from Google in January $2010^{6}$ showed Unicode support across the web nearing 50\% and growing quickly, this alone represents a challenge to local language TTS that may be easily overlooked. Even with the development of high quality, easily accessible regional language TTS engines and screen readers, these tools are only as good as the material they are trying to access.

\section{Discussion}

Our initial interviews that would lead us to creating the two surveys we deployed had already indicated that the JAWS vs. NVDA question was likely to be central to any discussion of the future of screen readers in India. The importance of this question is only reinforced by the findings from the survey.

JAWS remains by far the dominant screen reader in the world, holding over $67 \%$ of the market share. ${ }^{7}$ In our own sample, almost all the interviewed expert users had JAWS as their primary screen reader, even when they considered themselves advanced NVDA users. Yet, it is not clear that this dominance will remain the case perpetually. NVDA has only existed for a fraction of the time JAWS has, and is gaining ground quite rapidly. The WebAIM surveys show that there has been a growing move towards open source software use among screen readers around the world, with NVDA the fastest gaining application in the screen reading category, currently outranked only by JAWS in terms of the number of installed machines worldwide.

As we have pointed out here, there are both business-related and technical reasons for why open source software may make a very strong impact starting in the developing world. NVDA's website lists the Mozilla Foundation, Adobe, Yahoo!, and Microsoft as significant donors to the project ${ }^{8}$, in addition to which there have been a number of firms encouraging NVDA use in place of pirated software on their machines, and in doing so, building

\footnotetext{
${ }^{6}$ http://googleblog.blogspot.com/2010/01/unicode-nearing-50-ofweb.html

${ }^{7}$ http://webaim.org/projects/screenreadersurvey3/

${ }^{8}$ http://www.nvaccess.org/blog
}

support for their internal applications on NVDA. Online forums show that there are regular updates of new applications being supported or minor adjustments to improve the product occurring on a regular basis.

One of the features that made NVDA particularly attractive to users was that it can be used with a USB stick without the need for any further software.

"With JAWS, because it has to load its video training manager, you can't load it through a USB stick... The portable version of NVDA, especially for people who are going around in cybercafés and using computers of their friends, or on the university campus, or anywhere like that, it's definitely a big advantage that you can easily just stick in your thumb drive and start using NVDA on the move."

Dr. Homiyar Mobedji, Mumbai

Given that the vast majority of vision impaired computer users in India do not have access to their own machines, this is quite important, though not something one foresees competitors ignoring for long. It is, however, the apparent momentum surrounding NVDA that may be its strongest advantage.

NVDA's funding model has an "OSS 2.0" flavor to it [16]. That is, while the older OSS model was personified by Linux, Perl, etc., each of which had many contributors and little explicit organization, the newer OSS 2.0 model has few developers receiving feedback from a larger community or users, often receiving funding from outside sources. NVDA does exactly this - though OSS 2.0 is also described largely as being for-profit with value-added services such as Red Hat or IBM's Star Office (compared with totally free OpenOffice.org).

Besides the general support from the online community, there have been a number of proactive steps that have pushed the development of NVDA further. First, a significant number of the development team for SAFA (Screen Access For All), an Indian language screen reader, were reassigned to work on NVDA (according to personal communication with Dipendra Manocha, coordinator for the DAISY Consortium). The Saksham Trust, a New Delhi-based NGO, recently gave away 200 netbooks with NVDA pre-installed to visually impaired persons "who want to use the computers for their daily reading and writing purpose, but due to the high cost of hardware and software, they are unable to do so."9

Finally, the possibility of local language development has the potential to be a very important factor as time goes by. While the reality of the day is that computer users in India can manage with (and probably prefer) English language interfaces, this is likely to change in time, and the day we see screen readers with high quality Hindi, Telugu, or Bangla output may not be far.

For commercial screen readers like JAWS, the situation is not necessarily ominous; these findings may only mean that business models for these markets be different. As in the case of other major software firms, hard questions on pricing strategies for international markets will have to be dealt with, and in an increasingly competitive market that corresponds to the ability to

\footnotetext{
${ }^{9}$ http://www.saksham.org/project.php?id=11
} 
support custom applications. This may require that commercial screen readers expand their developer networks.

Finally, our study on screen readers also points to directions ahead for major software and web service companies or operating systems. For instance, one of the most frequent complaints of NVDA users was the lack of appropriate support for office applications. Interestingly, Microsoft, with its own OS-based screen reader, Narrator, may also be an important player in this space over time (only four respondents regularly used the Windows-based Narrator, the same number that used the Linuxbased Orca)

"NVDA and JAWS comparatively, JAWS will work with many more applications... especially the formal ones like Word, with Microsoft Office." Dr. Homiyar Mobedji,
Mumbai

\section{Conclusion}

Assistive technology use related to vision impairment in India (or in the developing world broadly) has had very limited serious scholarly interest until recently. Our purpose in this study was to highlight some of the important issues around screen reader use in India in the hope that in time the subject becomes an important enough area of concern within the rapidly growing ICTD community.

A number of the findings here give us an insight into how screen readers are being adopted in a country where the proliferation of assistive technology within the population with vision impairments is still low, and in time as research in this space expands, we will be able to build on some of the findings highlighted here.

For one, the issues on piracy that we highlight are likely to have deep impacts on the market for screen readers, and as we discuss in section 4, potentially spur an investment into NVDA or other open source screen readers in accordance with the OSS 2.0 model of development.

The two instances in our study where we find the most significant findings also hold potential for follow-up work. The findings around cost of software switching from dominant software to open source software give us an area of further investigation that can be applied not just to screen reading, but more broadly to a range of other software applications. Finally, our findings on the surface factors relating to screen reader preference need to be validated with screen reader use patterns in other parts of the world. Through this, we hope the conclusions of this work are relevant not just to assistive technology in the developing world, but to the wider community of AT users across the globe.

\section{REFERENCES}

[1] Pal, J., U. Vallauri, and V. Tsaran. Low-cost assistive technology in the developing world: a research agenda for information schools. 2011. ACM.

[2] Osorio, C.A., A Contribution to the Understanding of Illegal Copying of Software. Levine's Working Paper Archive, 2003.

[3] Maskus, K.E., Intellectual property challenges for developing countries: An economic perspective. University of Illinois Law Review, 2001: p. 457.

[4] Katz, M.L. and C. Shapiro, Technology adoption in the presence of network externalities. The journal of political economy, 1986: p. 822-841.

[5] Huysmans, P., K. Ven, and J. Verelst, Reasons for the nonadoption of OpenOffice. org in a data-intensive public administration. First Monday, 2008. 13(10).

[6] Kelley, J., R. Patnayakuni, and Z. Xiang, " OOo! I want that!" An Examination of Individual Adoption of OpenOffice Software. AMCIS 2006 Proceedings, 2006: p. 109.

[7] Abdelwahab, M.M.A., Assessing the switching barriers between Microsoft Office and OpenOffice. org, 2009, Carleton University.

[8] Himanen, P., L. Torvalds, and M. Castells, The hacker ethic2002: Random House Trade Paperbacks.

[9] Grant, R., Ubuntu Linux for non-geeks: a pain-free, projectbased, get-things-done guidebook2006: No Starch Press, San Francisco.

[10] Lakhani, K. and R. Wolf, Why hackers do what they do: Understanding motivation and effort in free/open source software projects. MIT Sloan Working Paper No. 4425-03, 2003.

[11] Bagozzi, R.P. and U.M. Dholakia, Open source software user communities: A study of participation in Linux user groups. Management Science, 2006. 52(7): p. 1099.

[12] Pal, J., The developmental promise of information and communications technology in India. Contemporary South Asia, 2003. 12(1): p. 103-119.

[13] Tucker, R. and K. Shalonova. Supporting the creation of TTS for local language voice information systems. 2005.

[14] Weber, F. and K. Bali. Real Voice and TTS Accent Effects on Intelligibility and Comprehension for Indian Speakers of English as a Second Language. 2009.

[15] Raghavendra, E.V. and K. Prahallad. A multilingual screen reader in Indian languages. 2010. IEEE.

[16] Fitzgerald, B., The transformation of open source software. Mis Quarterly, 2006. 30(3): p. 587-598. 